CORRECTIONS

\title{
David Morrell
}

This Obituary for David Morrell (BMJ 2012;344:e3227, doi:10. 1136/bmj.e3227) incorrectly stated that the MSc starting in 1986 at London was the first in general practice. In fact, the first such MSc started at Glasgow in 1982, followed by those at Exeter and Leeds in 1983. The Exeter MSc was the first to be multidisciplinary.

Cite this as: BMJ 2012;344:e3917

๑ BMJ Publishing Group Ltd 2012 